\title{
Co-evolution with Staphylococcus aureus leads to lipopolysaccharide alterations in Pseudomonas aeruginosa
}

\author{
Mikael Tognon ${ }^{1,2,5}$, Thilo Köhler ${ }^{1,2,5}$, Bartosz G Gdaniec ${ }^{1,2}$, Youai Hao ${ }^{3}$, Joseph S Lam³, \\ Marie Beaume ${ }^{1,2}$, Alexandre Luscher ${ }^{1,2}$, Angus Buckling ${ }^{4}$ and Christian van Delden ${ }^{1,2}$ \\ ${ }^{1}$ Transplant Infectious Diseases Unit, University Hospitals of Geneva, Genève, Switzerland; ${ }^{2}$ Department of \\ Microbiology and Molecular Medicine, University of Geneva, Genève, Switzerland; ${ }^{3}$ Department of Molecular \\ and Cellular Biology, University of Guelph, Guelph, ON, Canada and ${ }^{4}$ Environment and Sustainability \\ Institute, University of Exeter, Penryn, UK
}

\begin{abstract}
Detrimental and beneficial interactions between co-colonizing bacteria may influence the course of infections. In cystic fibrosis (CF) airways, Staphylococcus aureus prevails in childhood, whereas Pseudomonas aeruginosa progressively predominates thereafter. While a range of interactions has been identified, it is unclear if these represent specific adaptations or correlated responses to other aspects of the environment. Here, we investigate how $P$. aeruginosa adapts to $S$. aureus by evolving $P$. aeruginosa in the presence and absence of $S$. aureus. $P$. aeruginosa populations that evolved for 150 generations were sequenced and compared to the ancestor strain. Mutations in the Wsp signaling system were identified in both treatments and likely occurred because of low oxygen availability. Despite showing increased killing activity, wsp mutants were less fit in the presence of $S$. aureus. In contrast, mutations in lipopolysaccharide (LPS) biosynthesis occurred exclusively in cocultures with $S$. aureus and conferred a fitness gain in its presence. Moreover, they increased resistance towards beta-lactam antibiotics. Strikingly, both mutations in wsp and LPS genes are observed in clinical isolates from CF-patients. Our results suggest that $P$. aeruginosa LPS mutations are a direct consequence of $S$. aureus imposed selection in vitro.
\end{abstract}

The ISME Journal (2017) 11, 2233-2243; doi:10.1038/ismej.2017.83; published online 26 May 2017

\section{Introduction}

Pseudomonas aeruginosa is an opportunistic human pathogen responsible for acute and chronic infections in immunocompromised hosts. In cystic fibrosis (CF) patients, $P$. aeruginosa establishes chronic respiratory infections leading to progressive decrease of lung function (Folkesson et al., 2012), ultimately requiring lung transplantation. $P$. aeruginosa adapts to the CF-lung and is difficult to eradicate because of its ability to resist antimicrobial treatments. Part of the adaptation to the CF-environment involves the loss or downregulation of virulence factors including lipopolysaccharide (LPS), quorum sensing (QS) regulators and siderophore receptors as well as increased antibiotic resistance (Folkesson et al., 2012). A key question to address is what is driving

Correspondence: C van Delden, Transplant Infectious Diseases Unit, Department of Medical Specialties, University Hospitals of Geneva, 4, Rue Gabrielle-Perret-Gentil, CH-1211 Genève 14, Switzerland.

E-mail: christian.vandelden@hcuge.ch

${ }^{5}$ These two authors contributed equally to this work.

Received 5 September 2016; revised 24 March 2017; accepted 19 April 2017; published online 26 May 2017 these evolutionary changes. While the host environment and treatment strategies likely play a fundamental role (Folkesson et al., 2012), interactions with conspecifics (Köhler et al., 2009) and other coinfecting species may be critical (Mashburn et al., 2005).

P. aeruginosa colonizes up to $80 \%$ of adult CFpatients, while the dominant bacterial species in children and young adults is Staphylococcus aureus with a prevalence of up to $75 \%$ (Cystic Fibrosis Foundation Patient Registry, 2015). Crucially, P. aeruginosa and $S$. aureus frequently co-inhabit the CF lungs, suggesting that some of the $P$. aeruginosa adaptations may be driven by interactions with $S$. aureus. Consistent with this view, several QScontrolled strategies are used by $P$. aeruginosa to outcompete $S$. aureus in vitro: (i) lysis of the $S$. aureus cell wall by the secreted LasA elastase (Mashburn et al., 2005), (ii) inhibition of the $S$. aureus respiratory chain by 4-hydroxy-2-heptylquinoline- $N$-oxide (HQNO) (Hoffman et al., 2006; Kim et al., 2015), and (iii) dispersal of $S$. aureus biofilms by rhamnolipids (Abdel-Mawgoud et al., 2010; Diaz De Rienzo et al., 2016). However, these studies contrast with others that show beneficial 
interactions between the two pathogens. For instance, $P$. aeruginosa protects $S$. aureus from Dictyostelium discoideum phagocytosis (Yang et al., 2011), and increases the survival of S. aureus during antibiotic treatments (Baldan et al., 2014; DeLeon et al., 2014). In addition, clinical strains of $P$. aeruginosa isolated from the lungs of adult CFpatients are impaired in their ability to outcompete $S$. aureus, but rather interact in a commensal-like fashion (Michelsen et al., 2014; Frydenlund Michelsen et al., 2016). These data illustrate a complex relationship between $P$. aeruginosa and $S$. aureus and call into question whether these phenotypic interactions are the result of selection imposed by $S$. aureus or simply correlated responses to other selection pressures.

One way to identify the potential consequences of selection imposed by $S$. aureus on $P$. aeruginosa is to conduct controlled in vitro evolution experiments (Harrison et al., 2008), and this is the approach we took in this study. Our in vitro set up was designed to mimic aspects of an in vivo situation, where a lowdensity $P$. aeruginosa population encounters a preestablished high-density $S$. aureus community. We evolved $P$. aeruginosa for over as estimated 150 generations in the presence or absence of $S$. aureus, with the $S$. aureus population renewed after each cycle. Surprisingly we found that wsp mutants, characterized by small, wrinkly colonies of $P$. aeruginosa, which showed increased killing activity towards $S$. aureus evolved in both treatments, demonstrating this interaction to be a correlated response to selection imposed by the abiotic conditions. However, LPS-deficient mutants evolved in the presence but not the absence of $S$. aureus, and conferred a fitness advantage. These mutants were also more resistant to beta-lactam antibiotics. Lipopolysaccharide mutants with both phenotypes are frequently observed in $P$. aeruginosa CF-isolates, suggesting that exposure to $S$. aureus might play a role in the in vivo adaptation of $P$. aeruginosa to the CF lung-environment.

\section{Material and methods}

M14 medium composition

An M9 salt $\left(\mathrm{Na}_{2} \mathrm{HPO}_{4} 6 \mathrm{gl}^{-1} ; \mathrm{KH}_{2} \mathrm{PO}_{4} 3 \mathrm{gl}^{-1} ; \mathrm{NaCl}\right.$ $0.5 \mathrm{~g} \mathrm{l}^{-1} ; \mathrm{NH}_{4} \mathrm{Cl} 1 \mathrm{gl}^{-1}$ ) base (Rudin et al., 1974) was modified to obtain similar growth rates for $S$. aureus and $P$. aeruginosa under static microtiter plate growth conditions. This medium, termed M14, was supplemented with $10 \mathrm{gl}^{-1}$ casamino acids (BD, Franklin Lakes, NJ, USA), magnesium sulfate $\left(\mathrm{MgSO}_{4}\right) 1 \mathrm{~mm}$, $2 \mathrm{mg} \mathrm{l}^{-1}$ thiamine (vitamin B1), $2 \mathrm{mg} \mathrm{l}^{-1}$ niacin (vitamin B3), $2 \mathrm{mg} \mathrm{l}^{-1}$ calcium pantothenate (vitamin B5), $0.1 \mathrm{mg} \mathrm{l}^{-1}$ biotin (vitamin B9) and $2 \mathrm{~g} \mathrm{l}^{-1}$ glucose.

Strains, plasmids and culture conditions

$P$. aeruginosa PA14 and $S$. aureus Newman strains used in this study are listed in Supplementary
Table S1. Plasmids and primers are listed in Supplementary Tables S2 and S3. P. aeruginosa and $S$. aureus were freshly grown each day on M14 agar plates overnight at $37^{\circ} \mathrm{C}$ if not stated otherwise. Bacterial suspensions were prepared in $0.9 \%(\mathrm{w} / \mathrm{v})$ $\mathrm{NaCl}$ from overnight cultures on M14 agar plates. All experiments were performed in microtiter plates using a multimode plate reader (Synergy H1 BioTek, Franklin Lakes, NJ, USA). Run and reading parameters were as follows: incubation at $37^{\circ} \mathrm{C}$ and linear shaking for 1 min before reading the optical density at $600 \mathrm{~nm}$ and fluorescence (excitation wavelength: $485 \mathrm{~nm}$, emission wavelength: $528 \mathrm{~nm}$ ) every hour for $24 \mathrm{~h}$. All experiments were performed in technical triplicates.

\section{Evolution experiment}

The evolution experiment was performed in 96-well microtiter plates using liquid M14 medium as illustrated in Figure 1. Based on $P$. aeruginosa growth in our experimental conditions (Supplementary Figure S1), we calculated the number of generations $(n)$ by $n=\left(\operatorname{logCFU~} t_{24}-\log C F U t_{0}\right) / \log 2$. Taking into account the linear growth pattern of $P$. aeruginosa, the initial bacterial concentration of $10^{6} \mathrm{CFU} \mathrm{ml} \mathrm{m}^{-1}$ at $t_{0}$ and the final bacterial concentration of $10^{9} \mathrm{CFU} \mathrm{ml}{ }^{-1}$ at $t_{24}$ we calculated $n=9.96$ for one $24 \mathrm{~h}$ cycle. Evolution of $P$. aeruginosa in the presence or absence of $S$. aureus was achieved by performing 15 (24-h) cycles of incubation (15 serial transfers) within pre-incubated cultures of $S$. aureus or sterile M14 medium, respectively. On the first day, $5 \times 10^{5}$ CFU of $P$. aeruginosa were used to inoculate each well of the plate. Pre-established cultures of $S$. aureus were prepared each day from fresh overnight cultures using $10^{7} \mathrm{CFU}$ of $S$. aureus, and were incubated for $6 \mathrm{~h}$ at $37^{\circ} \mathrm{C}$ without shaking before introduction of $P$. aeruginosa. To ensure a steady $P$. aeruginosa to $S$. aureus ratio between $1: 1000$ and $1: 100$, cultures were diluted before their transfer to the next incubation plate. In order to transfer a $P$. aeruginosa CFU range of $10^{5}-10^{6}$ (bacterial concentration of $10^{6}-10^{7} \mathrm{CFU} \mathrm{ml}^{-1}$ ), $20 \mu \mathrm{l}$ of the cultures were withdrawn and diluted $1000 \times$ in $0.9 \%(\mathrm{w} / \mathrm{v}) \mathrm{NaCl}$. Ten microliters of the $1000 \times-$ diluted cultures were then used to inoculate the next plate for a new 24-h round of incubation. Staphylococcus aureus cells could not be separated from $P$. aeruginosa cells during the transfer. Viable plate counts after each 24-h cycle allowed us to follow the survival of each species throughout the co-evolution assay (Supplementary Figure S2). Culture samples were stored at the end of each $24 \mathrm{~h}$ cycle at $-80^{\circ} \mathrm{C}$.

Whole genome sequencing and bioinformatics analysis Bacterial gDNA was extracted from the whole evolved populations (triplicate samples) and sequenced on an Illumina HiSeq 2500 platform (Fasteris SA, Plan-les-Ouates, Switzerland) generating 100-bp paired end reads to obtain an average of 

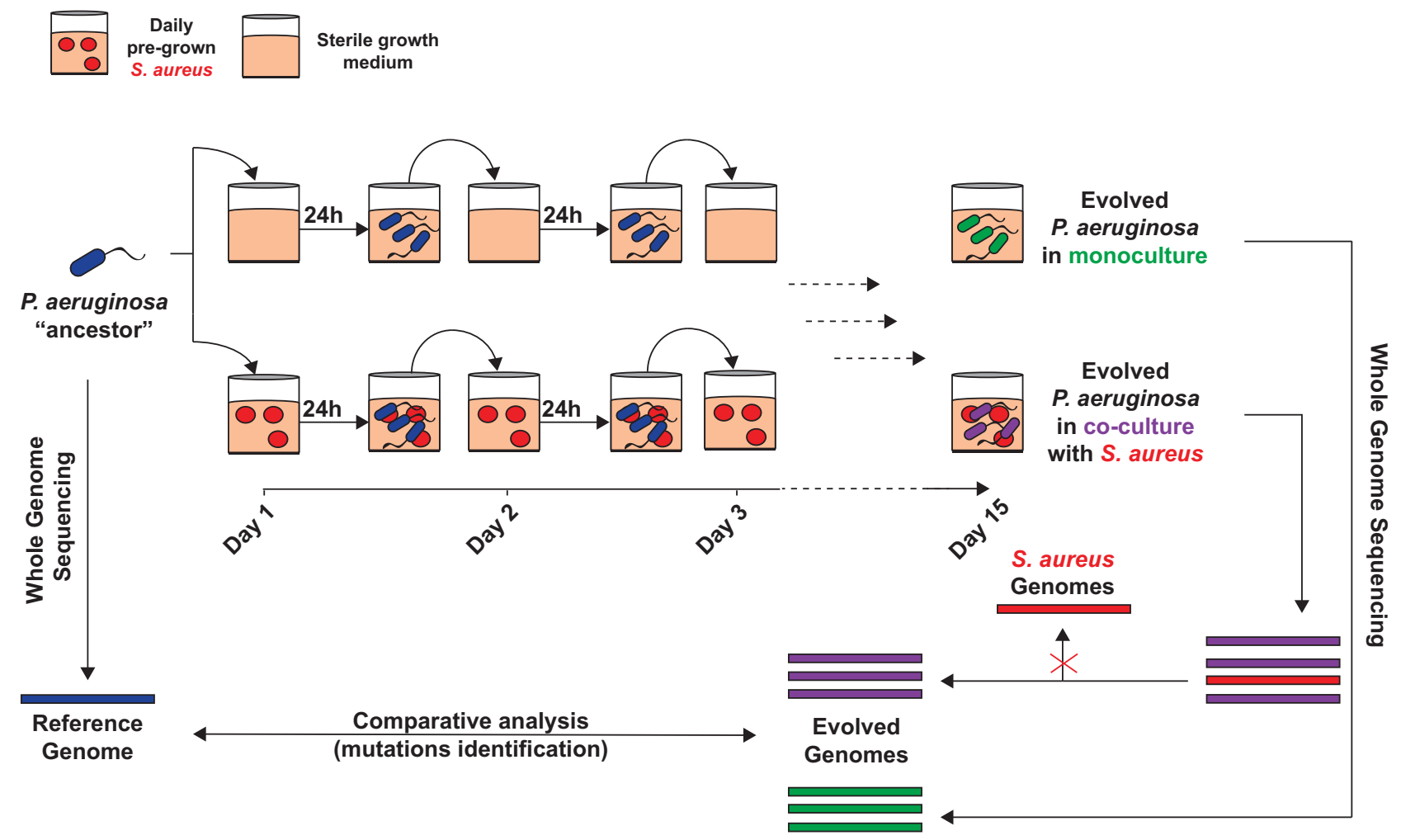

Figure 1 Evolution experiment setup. The ancestor strain of $P$. aeruginosa was evolved by performing 15 cycles of $24 \mathrm{~h}$ incubation each with and without the presence of pre-incubated $S$. aureus cultures. At the 15th day, the resulting evolved genomes of $P$. aeruginosa descendants were sequenced and compared to the ancestor for the identification of mutations.

9187682 reads (range of 70667874-10707490 reads). The software SGA (Simpson and Durbin, 2010) was used to filter the reads and to assemble genomes. Reads were processed as follows: the adapter sequence (5'-AGATCGGAAGAGC-3') was removed at the end of the reads, and the end of the reads with a quality score below three were trimmed. Reads were discarded if the resulting length after trimming was below 49 bp. SGA was used to correct sequencing errors by searching for 27-mers with low frequency. Genomes were de novo assembled and specific 27-mers were mapped to the assembled contigs using SGA. The contigs were then mapped for each gene feature to the reference genome of P. aeruginosa PA14 RefSeq genome NC_008463.1 using Burrows-Wheeler Alignment Tool to create BAM files (Li and Durbin, 2010). The contigs were screened manually using IGV browser software (http://wwwbroadinstitute.org/igv) and blasted (NCBI Blast) to identify single nucleotide polymorphisms (SNPs), insertions/deletions (InDels) and rearrangements.

\section{Killing assays}

Killing assays were performed on $S$. aureus cells pregrown for $6 \mathrm{~h}$ in M14 medium under static growth conditions in microtiter plates. After the $6 \mathrm{~h}$ incubation, $100 \mu \mathrm{l}$ of $S$. aureus culture was removed and replaced with either $100 \mu \mathrm{l}$ M14 medium or
$P$. aeruginosa supernatant. Supernatants were obtained after $24 \mathrm{~h}$ growth in M14 medium, centrifugation at $3,800 \mathrm{~g}$ for $5 \mathrm{~min}$ at $4^{\circ} \mathrm{C}$ and subsequent filtration (0.22 $\mu \mathrm{m}$ Millipore filters). If required, $P$. aeruginosa supernatants were stored at $-20^{\circ} \mathrm{C}$. Growth (increase in $\mathrm{OD}_{600}$ ) was monitored in a plate reader (Biotek Synergy H1) for $24 \mathrm{~h}$. At the end of the killing assay, viable plate counts were performed to determine $S$. aureus survival.

\section{Fitness assays}

The fitness assays were performed using the protocol developed by Wiser and Lenski in a long-term evolution experiment (Wiser and Lenski, 2015). Isogenic mutants constructed from the ancestor strain of $P$. aeruginosa were used to assess their fitness in the presence and absence of $S$. aureus. The fitness assay was performed using $5 \times 10^{5}$ CFU of $P$. aeruginosa test strain and $5 \times 10^{5}$ CFU of $P$. aeruginosa ancestor mixed within either sterile M14 medium or with pre-incubated $S$. aureus cultures following the same protocol as for the evolution experiment. Viable plate counts were performed as described above. All assays were done in biological duplicates.

\section{LPS analysis}

Lipopolysaccharide preparation was performed as described previously (Hao et al., 2015; 
Wang et al., 2015). Lipopolysaccharide samples were analyzed by sodium dodecyl sulfate-polyacrylamide gel electrophoresis and visualized by silver staining (Hao et al., 2015; Wang et al., 2015).

\section{Minimal inhibitory concentration}

To determine antibiotic susceptibility, minimal inhibitory concentrations were performed in triplicates by two-fold serial dilutions in Müller-Hinton medium (Becton Dickinson, Franklin Lakes, NJ, USA) according to Clinical and Laboratory Standard Institute (CLSI) guidelines.

\section{Results}

Whole genome sequencing of evolved isolates

At the end of the evolution experiment (approximately 150 generations), the ancestor strain and the triplicate samples from $P$. aeruginosa populations, evolved with and without $S$. aureus, were submitted to whole genome sequencing. We identified two mutated genetic loci at high frequencies in all three $P$. aeruginosa monoculture replicates. The first locus was $\operatorname{wsp} A$, coding for a methyl-accepting chemotaxis-like transducer, and the second was lasR, coding for a QS transcriptional regulator. The mutations were either deletions (replicate A) or SNPs (replicates B and C) (Table 1). The mutations in $w s p A$ were all located in the predicted methyl-accepting domain of WspA (Figures 2a and b), whereas those in lasR were located in the C-terminal DNA-binding domain of LasR. While the wspA mutation was present in $100 \%$ of the evolved genomes and therefore likely fixed within the population, the lasR mutation was detected in only $30-40 \%$ of genomes at the end of the experiment.

Among the $P$. aeruginosa populations evolved in the presence of $S$. aureus, we identified five to six mutations in each of the triplicates (17 in all) at the end of the experiment. The diversity of targeted genes was higher than in the monoculture, but the majority fell within two functional categories: cell signaling (5/12) and cell membrane biosynthesis (7/12). As in the mono-cultures, mutations in the cell signaling category occurred in the Wsp signaling system, but targeted the $w s p E$ or the $w s p F$ gene (Figures 2a and b). Mutations in the cell membrane biosynthesis category occurred in orf $N$ and PA14_23400, two uncharacterized ORFs encoded in an LPS biosynthesis gene cluster, as well as in $\operatorname{dnp} A$ encoding a putative de- $N$-acetylase (Table 1). The majority of mutations in orfN, PA14_23400 and $\operatorname{dnp} A$ were either deletions or insertions, expected to result in loss of function of the corresponding proteins. These mutations occurred exclusively in clones retrieved from the co-cultures; a strong argument for a specific adaptation to the presence of $S$. aureus. The mutations in wspE (SNP) and wspF (SNP/deletion) occurred in the corresponding

Table 1 Whole genome sequencing data for evolved P. aeruginosa populations

\begin{tabular}{|c|c|c|c|c|c|c|c|}
\hline $\begin{array}{l}\text { Sample } \\
\text { name }\end{array}$ & $\begin{array}{l}\text { Gene name in } \\
\text { PA14 }\end{array}$ & $\begin{array}{l}\text { Functional } \\
\text { category }\end{array}$ & $\begin{array}{l}\text { Mutation } \\
\text { type }\end{array}$ & $\begin{array}{l}\text { InDel } \\
\text { (nts) }\end{array}$ & Mutation & $\begin{array}{l}\text { Mutation }{ }^{\mathrm{a}} \\
\text { tolerated }\end{array}$ & $\begin{array}{l}\text { Relative abundance } \\
\text { in population (\%) }\end{array}$ \\
\hline \multirow[t]{2}{*}{ M15A } & $w s p A$ & Cell signaling & Del & $\Delta 84$ & S285S & No & 100 \\
\hline & lash & Transcription & Del & $\Delta 17$ & X17 & - & 40 \\
\hline \multirow[t]{2}{*}{ M15B } & $w \operatorname{sp} A$ & Cell signaling & SNP & - & $\mathrm{A} 438 \mathrm{~V}$ & No & 100 \\
\hline & lasR & Transcription & SNP & - & $\mathrm{T} 222 \mathrm{I}$ & No & 30 \\
\hline \multirow[t]{2}{*}{ M15C } & $w s p A$ & Cell signaling & SNP & - & A418V & No & 100 \\
\hline & lasR & Transcription & SNP & - & C188R & No & 40 \\
\hline \multirow[t]{5}{*}{ C15A } & $w s p F$ & Cell signaling & SNP & - & M307V & No & 92 \\
\hline & $\operatorname{orf} N$ & LPS biosynthesis & In & +1 & X99 & - & 45 \\
\hline & orf $N$ & LPS biosynthesis & In & +2 & $\mathrm{X} 4$ & - & $<1$ \\
\hline & $\operatorname{dnp} A$ & LPS biosynthesis & Del & $\Delta 20$ & $\mathrm{X} 2$ & - & 4 \\
\hline & $\operatorname{mexI}$ & $\begin{array}{l}\text { Defense } \\
\text { mechanisms }\end{array}$ & SNP & - & G195E & No & $<1$ \\
\hline \multirow[t]{5}{*}{ C15B } & PA14_65570 & Unknown & Del & $\Delta 9$ & L173A & No & 59 \\
\hline & $W s p E$ & Cell signaling & SNP & - & A749V & No & 33 \\
\hline & $\operatorname{orf} N$ & LPS biosynthesis & Del & $\Delta 1$ & $\mathrm{X} 7$ & - & 52 \\
\hline & pilT & Cell signaling & Del & $\Delta 1365$ & $\begin{array}{c}\text { Gene } \\
\text { deleted }\end{array}$ & No & 50 \\
\hline & PA14_05160 & Unknown & Del & $\Delta 21$ & $\Delta 7$ аа & - & 50 \\
\hline \multirow[t]{7}{*}{ C15C } & $w s p F^{-}$ & Cell signaling & Del & $\Delta 41$ & X129 & - & 30 \\
\hline & PA14_23400 & Unknown & Del & $\Delta 1$ & $\mathrm{X} 22$ & - & 41 \\
\hline & $\operatorname{orf} N^{-}$ & LPS biosynthesis & Del & $\Delta 1$ & $\mathrm{X} 7$ & - & 16 \\
\hline & PÁ14_23400 & Unknown & SNP & - & Q329K & No & 24 \\
\hline & $\operatorname{orfN}$ & LPS biosynthesis & SNP & - & $\widehat{D} 249 G$ & No & 25 \\
\hline & $w s p F$ & Cell signaling & SNP & - & A160A & Yes & 24 \\
\hline & $\operatorname{dnp} A$ & LPS biosynthesis & Del & $\Delta 20$ & $\mathrm{X} 2$ & - & 2 \\
\hline
\end{tabular}

Abbreviations: $\Delta$, deletion of nucleotides; +, insertion of nucleotides; fs, frameshift; LPS, lipopolysaccharide; SNP, single nucleotide polymorphism; X, stop codon.

Co-culture samples from the 15th day are designated as C15, monoculture samples from the 15 th day as M15.

aas predicted by the SIFT algorithm (http://sift.jcvi.org/). 
protein domains involved in signal reception (WspE) and methylesterase activity (WspF). Phenotypes associated with these mutations will be described below. Additional mutations occurred in the mexI gene belonging to the mexGHI-opmD efflux operon $(<1 \%$ in replicate $\mathrm{A})$ and in the pilT gene involved a

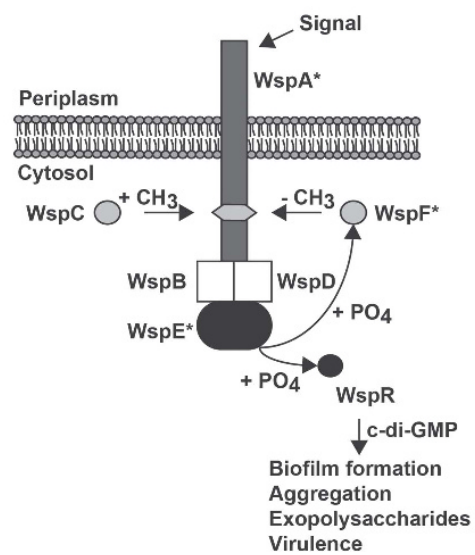

c
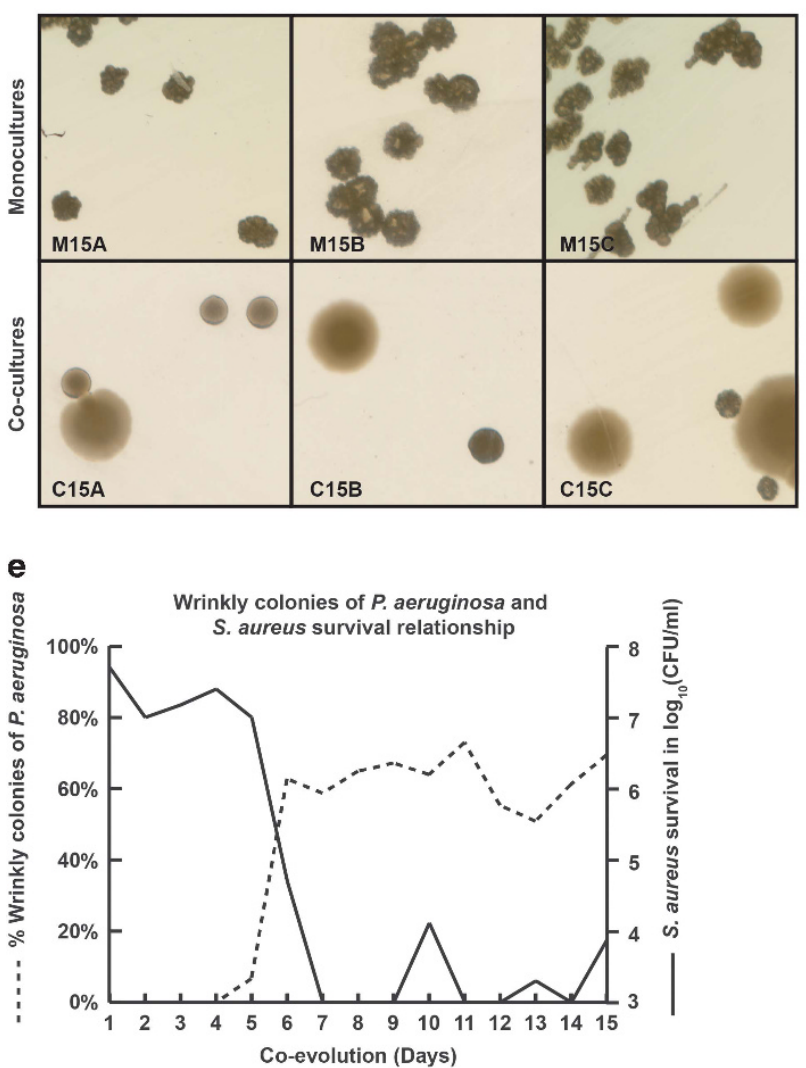

b
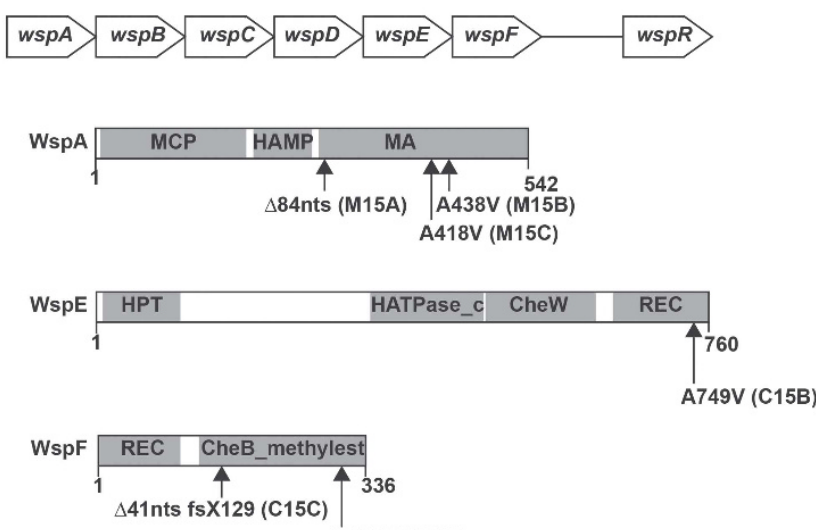

M307V (C15A)

d
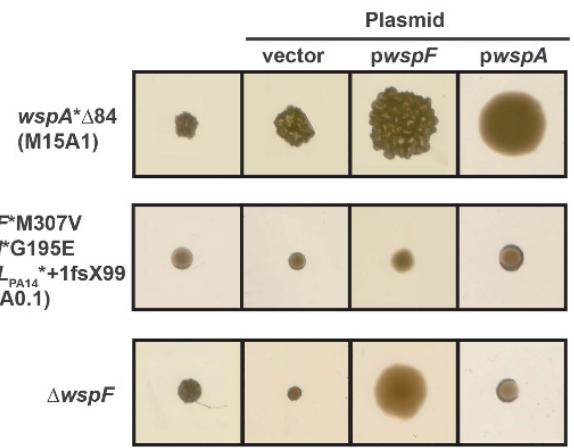

Ancestor

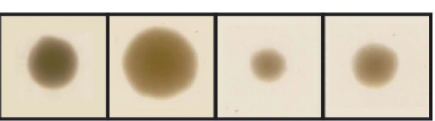

$\mathbf{f}$

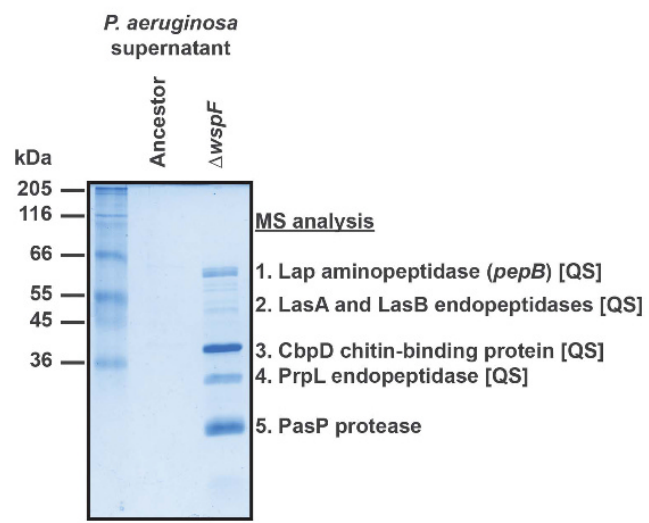

Figure 2 Scheme of the Wsp signaling system adapted from Hickman et al. (2005). Mutations identified in the evolution experiment are marked by asterisks (a). The wsp operon organization is shown at the top. Mutations identified are located in the methyl-accepting domain (MA) of WspA, in the receiver domain (REC) of WspE and the methylesterase domain (CheBmethylest) of WspF. Sample replicates are indicated in parenthesis, fs: frameshift, X: stop codon, $\Delta$ : deletion of nucleotides, +: insertion of nucleotides (b). Small wrinkly colonies (SWC) of $P$. aeruginosa from mono- (top-panels) and co-culture (lower panels) replicates at the end (15th day) of the evolution experiment (c). Mutations in the Wsp system were associated with wrinkly colony phenotype, which was restored by genetic complementation with the corresponding wsp gene (d). Relative abundance of small wrinkly colonies (SWC) of $P$. aeruginosa (dotted line) and survival of $S$. aureus during the evolution experiment (solid line), data shown represent the mean of three replicates (standard error (s.e.) $<10 \%)$ (e). Twenty microliters of filtered culture supernatants collected from the ancestor and a $\Delta w s p F$ mutant were run on an SDS-PAGE gel and stained with Coomassie blue. The five most prominent protein bands were excised and analyzed by mass spectrometry (MS). Four of the five proteases are known to be regulated by quorum sensing (QS) (f). SDS-PAGE, sodium dodecyl sulfate-polyacrylamide gel electrophoresis. 
in pili synthesis regulation (50\% in replicate $B)$. We also noticed the absence of lasR mutations in $P$. aeruginosa isolated from the co-cultures, which might have been counter-selected by the presence of $S$. aureus. In contrast to the mono-cultures, the frequency of the identified mutations was highly variable in the populations (between 2 and 92\%) and none of them reached fixation, which could reflect the dynamic interactions between $P$. aeruginosa and $S$. aureus in the co-culture.

\section{Wsp-deficient mutants appear as small wrinkly} colonies and their abundance is negatively correlated with $S$. aureus survival

When compared to the ancestor morphotype, smaller colonies with wrinkled appearance emerged in all replicates of both mono- and co-cultures (Figure 2c). This change in morphotype was linked to mutations in $w s p A$ for the mono-culture and to $w s p E$ and $w s p F$ for the co-cultures (Figures 2c and d). The wrinkled phenotype reached $100 \%$ at the end of the experiment in the mono-cultures (100\% wspA mutants), while the proportion of small, wrinkled colonies was variable in the co-cultures (Figure 2c), and appeared to correspond to the observed variable proportions of $w s p F(30$ and $92 \%$ ) and $w s p E(33 \%)$ mutant genomes (Table 1). A similar wrinkled colony phenotype was reported for a $w s p F$ deletion mutant of $P$. aeruginosa strain PAO1 (Hickman et al., 2005). To confirm that the mutations in the Wsp system were responsible for this phenotype, we cloned the wild-type PA14 $w s p A$ and $w s p F$ alleles on a plasmid (pwspA and $\mathrm{p} w s p F$ ) and expressed them in wspA and $w s p F$ mutants from the last cycle (Supplementary Materials). As expected, the wild-type morphology was restored in the $\operatorname{wsp} A$ mutant in the presence of $\mathrm{p} w s p A$, but not $\mathrm{p} w s p F$. The $w s p F$ mutant, which also harbors mutations in mexI and $\operatorname{orfN}$, showed a wrinkly phenotype after $72 \mathrm{~h}$, and complementation with $\mathrm{pwspF}$ did not completely restore wild-type morphology, possibly due to the presence of the additional mutations (Figure 2d). We therefore constructed a deletion in the $w s p F$ gene in the ancestor strain. This $\Delta w s p F$ mutant displayed the wrinkled phenotype and could be complemented by pwspF (Figure 2d). Thus the wrinkled phenotype is associated with mutations in the wsp genes, which appeared both in the mono- and co-cultures.

To follow the survival of $P$. aeruginosa and $S$. aureus during the co-evolution assay, we monitored viable counts throughout the 15 cycles of coculture. We observed a significant decrease in $S$. aureus survival after 5 days of co-evolution. This decrease in viable $S$. aureus counts coincided with the appearance of wsp mutants and their relative abundance within the $P$. aeruginosa populations (Figure 2e). No effect on $P$. aeruginosa survival was observed at the same time point. We therefore hypothesized that the acquisition of wsp mutation increased the killing activity of $P$. aeruginosa towards S. aureus. To assess whether this killing activity was caused by secreted factors, we collected supernatants of the ancestor and the constructed $\triangle w s p F$ mutant and compared their protein contents by sodium dodecyl sulfate-polyacrylamide gel electrophoresis (Figure 2f). The $\Delta w s p F$ supernatants displayed a pattern of overexpression in several proteins that were identified by mass spectrometry as proteases (LasA, LasB, Lap, PrpL, PasP) and a chitin-binding protein CbpD (Figure 2f). Interestingly, the identified proteins, with the exception of PasP, are all QS regulated in P. aeruginosa (ArevaloFerro et al., 2003; Marquart et al., 2005).

Supernatants of wsp mutants display QS-dependent killing towards S. aureus and other Gram-positive and Gram-negative bacterial species

To verify that the $S$. aureus killing activity was present in the culture supernatants of the $P$. aeruginosa wsp mutants, we incubated stationary phase $S$. aureus cultures with filter-sterilized $P$. aeruginosa supernatants $(50 \% \mathrm{vol} / \mathrm{vol})$. Supernatants from $w s p A, w s p F$ and $w s p E$ mutants were able to decrease $S$. aureus viability by at least six orders of magnitude after $24 \mathrm{~h}$ incubation (Figure 3a). The culture supernatant from the constructed $\Delta w s p F$ mutant showed a similar level of killing activity, suggesting that the wsp mutations in the evolved $P$. aeruginosa isolates are responsible for this activity. There were no significant differences in $S$. aureus viability using a $0.9 \%(\mathrm{w} / \mathrm{v}) \mathrm{NaCl}$ solution, sterile M14 medium, and sterilized spent M14 medium from 24-h $S$. aureus or $P$. aeruginosa ancestor cultures (Supplementary Figure S3). Since the LasA protease has been identified as a staphylolytic protease (Kessler et al., 1993), we wondered whether increased LasA secretion by the wsp mutants could be responsible for the observed $S$. aureus killing. We therefore constructed a las $A$ deletion in the $\Delta w s p F$ mutant. Supernatants of this $\Delta w s p F \Delta$ las $A$ double mutant still showed $S$. aureus killing activity (Figure 3a). However, when the QS-regulatory gene lasR was deleted in the $\Delta w s p F$ strain, or altered by selection in the wspA mutant (wsp $A^{*} \Delta 84$ las $^{*} \Delta 17 \mathrm{fsX} 17$ ), the level of killing activity was dramatically decreased (Figure 3a). Since the three QS-systems show intricate interdependencies and overlaps in their target genes (Jimenez et al., 2012), we further investigated whether this killing activity was also affected by the Rhl and MvfR QS systems. We thus constructed deletions in $r h l R$ and $m v f R$ in the $\triangle w s p F$ strain background to obtain the double mutants $\Delta w s p F \Delta r h l R$ and $\Delta w s p F \Delta m v f R$. As shown in Figure 3a, deletion of $m v f R$ strongly reduced the killing activity, similar to a lasR mutant, while the rhlR mutation completely abolished killing activity. This suggests that factor(s) controlled by either of these three QS-systems are involved in $S$. aureus killing. To assess whether the killing activity was 

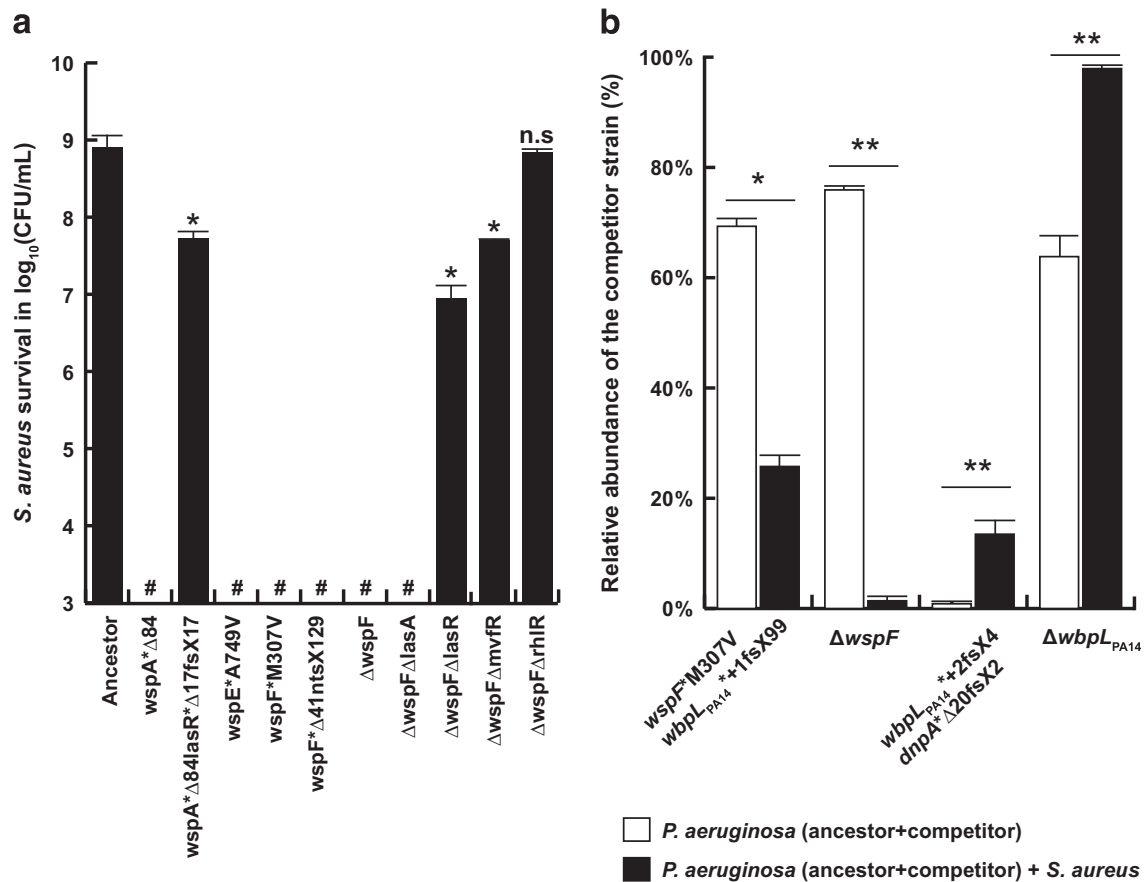

Figure 3 Killing activity of filter-sterilized P. aeruginosa supernatants on $S$. aureus cells. \#: below detection level (10 $\left.{ }^{3} \mathrm{CFU} \mathrm{ml}^{-1}\right)(\mathbf{a})$. Fitness assay comparing the ability of evolved and constructed $P$. aeruginosa mutants to invade the ancestor strain, starting from a 1:100 ratio, in the presence or absence of a pre-established $S$. aureus population (b). Statistical significance was determined using paired Student $t$-tests ( $: P<0.05,{ }^{* *}: P<0.01,{ }^{* * *}: P<0.001$; ns, not significant).

specific to the $S$. aureus Newman strain, we performed similar survival assays with different Gram-positive and Gram-negative bacterial species. A similar 6-log reduction in viable counts was observed with two different $S$. aureus strains (RN6390 (MSSA) and USA300 (MRSA)), whereas $\Delta w s p F$ supernatant reduced viable counts of lung commensal strains Actinomyces israelli and Neisseria flavescens by 3-4 logs. In contrast, growth inhibition, but only weak reduction in viable counts ( $<50$ fold) was observed with Klebsiella pneumonia, Rothia mucilaginosa and Staphylococcus epidermidis (Supplementary Figure S4).

Mutants in the Wsp system are adapted to the experimental growth conditions

Since mutations in the wsp system occurred in both mono- and co-cultures, we hypothesized that mutations in the Wsp system reflect an adaptation to our experimental set-up rather than to the presence of $S$. aureus. We therefore tested the fitness of $w s p F$ mutants, in the presence and absence of preestablished $S$. aureus populations, against the ancestor strain (Figure $3 \mathrm{~b}$ ). The proportion of $w s p F$ mutants increased from initially $1 \%$ to $>70 \%$ in the absence of $S$. aureus. In contrast, the proportion increased only to $30 \%$ in the $w s p F$ M307V mutant and did not increase in the constructed $\Delta w s p F$ knockout mutant in presence of $S$. aureus (Figure $3 \mathrm{~b}$ and Supplementary Figure S5), suggesting that a $w s p F$ mutant is less fit in the presence than in the absence of $S$. aureus.
During the co-evolution assay, we observed a pellicle at the liquid surface of the $w s p F$ mutants. This is reminiscent of the wrinkly spreader phenotype of $P$. fluorescens SBW25, which appears as an adaptation to oxygen limitation by forming cellular mats at the air-liquid interface, when grown under static conditions (Spiers et al., 2002, 2003; Goymer et al., 2006; Bantinaki et al., 2007). We wondered whether the wsp mutants resulted from a similar adaptation to our static microtiter plate assay conditions. We therefore repeated the co-evolution experiment over 5 days under both static and shaking growth conditions. Wrinkly colonies of $P$. aeruginosa appeared exclusively in static monocultures (data not shown). Two selected clones each harbored a different mutation in the methylesterase domain of WspF. Hence, we conclude that wsp mutants apparently occurred in response to oxygen limitation in our experimental set-up, and not as a selection imposed by $S$. aureus.

Mutations abolishing O-specific antigen LPS synthesis are selected in response to $S$. aureus

Mutations in $P$. aeruginosa that appeared specifically in the co-cultures were mainly classified within the cell wall/membrane synthesis category. Only the orf $N$ locus was mutated in all three replicates, representing $45 \%, 52 \%$ and $41 \%$ of the genomes in replicates A, B and C, respectively (Table 1). Closer analysis of the OrfN amino acid sequence revealed that it shares $60 \%$ identity to one of the glycosyltransferase $\mathrm{WbpL}$ of PAO1 involved in the initiation 
of the biosynthesis of the common polysaccharide antigen and the O-specific antigens (OSA) (Rocchetta et al., 1998). Furthermore, orf $N$ is located within the LPS biosynthesis gene cluster involved in $\mathrm{O} 19$ serotype synthesis of PA14. Based on these and the following data we will refer to orfN as $w b p L_{\mathrm{PA} 14}$. To confirm the role of $\mathrm{WbpL}_{\mathrm{PA} 14}$, we compared the LPS profiles of the ancestor and a constructed $\Delta w b p L_{\text {PA14 }}$ mutant by sodium dodecyl sulfate-polyacrylamide gel electrophoresis and silver staining (Figure 4). As expected, $\Delta w b p L_{\mathrm{PA} 14}$ showed impaired production of OSA. However, this defect could be restored by complementation with the cloned wild-type allele from PA14. Thus, loss of WbpL $\mathrm{PA}_{14}$ prevents the biosynthesis of the OSA side chain; hence only the fast migrating core LPS band was observed in the $\Delta w b p L_{\mathrm{PA14}}$ mutant (Figure 4 ).

We hypothesized that if $w b p L_{\mathrm{PA} 14}$ inactivation, and hence loss of OSA LPS, is a specific response to the

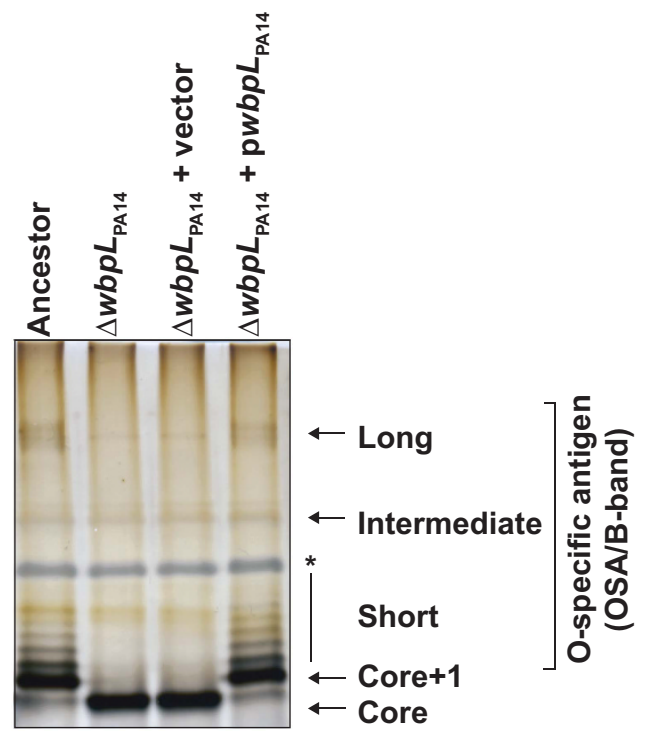

Figure 4 Lipopolysaccharide (LPS) profiles of $P$. aeruginosa ancestor strain and $w b p L_{\mathrm{PA} 14}$ mutant. The O-specific antigen LPS synthesis is absent in the $w b p L_{\mathrm{PA} 14}$ mutant. Asterisk indicates band corresponding to proteinase K. presence of $S$. aureus, we would observe an increased fitness of $w b p L_{\mathrm{PA} 14}$ mutants in the presence of the competitor. We therefore performed the same fitness assays as described above with the $w s p F$ mutant. Indeed, while the $w b p L_{\mathrm{PA}_{14}}-d n p A$ mutant remained at the initial 1\% ratio to the ancestor in the absence of $S$. aureus, the ratio increased to $12 \%$ in the presence of $S$. aureus (Figure 3b). Interestingly, the $w b p L_{\mathrm{PA} 14}$ mutations were detected between the third and fourth day of co-evolution (Supplementary Table S1), before the appearance of wsp mutations (Days 5-6). We therefore conclude that LPS loss is the main response of the adaptation of $P$. aeruginosa when co-cultured with $S$. aureus in our experimental setting.

The loss of $w b p L_{P A 14}$ increases the resistance of P. aeruginosa to beta-lactams

The loss of WbpLPA14 caused $P$. aeruginosa to produce a truncated version of LPS with only core oligosaccharide and devoid of OSA (Figure 4). We wondered whether this would affect the antibiotic susceptibility of the $w b p L_{\text {PA14 }}$ mutants. We performed antibiotic susceptibility tests by measuring minimal inhibitory concentrations on the ancestor, the evolved $w b p L_{\mathrm{PA} 14}{ }^{*} \mathrm{D} 249 \mathrm{G}$ single-mutant (C15C3) and a defined $\triangle w b p L_{\mathrm{PA} 14}$ mutant. Both the evolved and the constructed $w b p L_{\mathrm{PA}_{4}}$ mutants showed increased antibiotic resistance to beta-lactam antibiotics, but not to ciprofloxacin or polymyxin (Table 2). The antibiotic-resistance phenotype could be reversed by complementation with the cloned wild-type allele of $w b p L_{\text {PA14 }}$. These results showed that adaptation to $S$. aureus has led to selection of $w b p L_{\mathrm{PA} 14}$ mutants, which have improved fitness in the presence of $S$. aureus and displayed increased resistance to beta-lactam antibiotics.

\section{Discussion}

Evolution of $P$. aeruginosa in the presence and absence of $S$. aureus identified the Wsp signal transducing system (mono- and co-culture) and LPS

Table 2 Minimal inhibitory concentrations $\left(\mu \mathrm{g} \mathrm{ml}^{-1}\right)$ for $P$. aeruginosa strains

\begin{tabular}{|c|c|c|c|c|c|c|c|}
\hline \multirow[t]{2}{*}{ Antibiotic } & \multicolumn{7}{|c|}{ Strain } \\
\hline & Ancestor & C15C3 & $\begin{array}{l}\text { C15C3 } \\
\text { vector }\end{array}$ & $\begin{array}{l}\text { C15C3 } \\
p w b p L\end{array}$ & Ancestor $\Delta w b p L$ & $\begin{array}{c}\text { Ancestor } \Delta w b p L \\
\text { vector }\end{array}$ & $\begin{array}{c}\text { Ancestor } \Delta w b p L \\
p w b p L\end{array}$ \\
\hline Aztreonam & 4 & $16(\mathrm{R})$ & 8 & 4 & 8 & 8 & 4 \\
\hline Carbenicillin & 32 & 128 & NA & NA & 128 & NA & NA \\
\hline Cefepime & 2 & $8(\mathrm{R})$ & 4 & 2 & 4 & 4 & 2 \\
\hline Ceftazidime & 1 & 4 (S) & 2 & 1 & 2 & 4 & 1 \\
\hline Cefotaxime & 16 & $64(\mathrm{R})$ & 32 & 16 & 32 & 32 & 16 \\
\hline Meropenem & 0.125 & 0.125 (S) & 0.125 & 0.125 & 0.5 & 0.5 & 0.125 \\
\hline Piperacillin- & 2 & $8(\mathrm{~S})$ & 8 & 2 & 8 & 8 & 2 \\
\hline Tazobactam & & & & & & & \\
\hline Ciprofloxacin & 0.0625 & 0.0625 (S) & 0.0625 & 0.0625 & 0.0625 & 0.0625 & 0.0625 \\
\hline Polymyxin & 2 & $2(\mathrm{~S})$ & 2 & 4 & - & 2 & 4 \\
\hline
\end{tabular}

Abbreviations: NA, not applicable; R, resistant; S, susceptible according to the EUCAST breakpoints (2014). 
biosynthesis (co-culture) as the main targets. Parallel evolution in both mono- and co-culture samples suggests a strong selective pressure in our experimental setup. Mutations in the Wsp system resulted in a wrinkly colony morphology (Kirisits and Parsek, 2006; Haussler and Fuqua, 2013) and secretion of extracellular factor(s) able to kill $S$. aureus cells. The wbpL mutations that occurred only in the co-culture resulted in loss of OSA LPS-biosynthesis and conferred a fitness gain when co-cultured with $S$. aureus. These mutants were also more resistant towards beta-lactam antibiotics.

The Wsp system of $P$. aeruginosa transduces a signal through its inner membrane sensor WspA to WspE, which in turn phosphorylates both WspF, a negative regulator of the system, and the diguanylate cyclase WspR (Hickman et al., 2005). This results in increased amounts of the second messenger cyclicdi-GMP, which regulates the expression of several virulence factors (Guvener and Harwood, 2007) (Figure 1a). The mutations identified in our study in the methyl-accepting domain of WspA, the receiving domain of $\mathrm{WspE}$ and the methylesterase domain of WspF are expected to cause autoinduction of the Wsp signaling cascade. These mutations prevent the resetting of the system to the default state mediated by methylation of WspF, and phenotypically translates into the formation of wrinkled colonies. These findings are consistent with studies in Burkholderia cenocepacia, where evolution in static monocultures led to the selection of wsp mutants (Cooper et al., 2014; O'Rourke et al., 2015). Interestingly, a A407V substitution in the WspA protein of $B$. cenocepacia, corresponding to our A418V mutation, resulted in Wsp autoinduction. In our study, we presume that wsp mutants were selected because of a fitness gain imposed by limiting oxygen availability under static growth conditions. In agreement with wrinkly spreaders in Pseudomonas fluorescens, we propose that the increased production of Pel polysaccharide in wsp mutants of $P$. aeruginosa (Hickman et al., 2005) allows the attached cells to reach the airliquid interface. Since our wsp mutants were nonmotile the cell surface could not be reached by swimming motility (Supplementary Figure S6). The attached cells form a pellicle at the air-liquid interface where oxygen availability is optimal (Koza et al., 2011). Unexpectedly, the wsp mutants, whether selected in the mono- or the co-culture, displayed an impressive killing activity ( $>$ 6-log reduction), which was not limited to $S$. aureus. The killing activity was LasR-dependent, but independent of LasA protease activity. Since both RhlR and MvfR (Pqs) mutations also abolished the killing activity it seems that factor(s) co-regulated by these systems are involved in the $S$. aureus killing. These could include other proteases, HCN, pyocyanin or rhamnolipids. Interestingly, wsp mutants that show the same or similar mutational loci have been identified in $P$. aeruginosa isolates from chronically infected CF-patients (Smith et al., 2006; Marvig et al., 2013, 2015) and were associated with a poor prognosis (Starkey et al., 2009; Malone, 2015). Although selection of these mutations could happen for different reasons, similarities might exist between the growth conditions within the CF-lung and our experimental conditions.

Interestingly, lasR mutants emerged in the monocultures but not in the co-cultures. Under QSrequiring growth conditions, mutants in lasR emerge within a cooperator population. These are considered as cheaters, since they do not pay their share for the production of public goods. Since lasR is not required for growth in the M14 medium (data not shown), lasR mutations might have emerged simply to compensate the energy burden imposed by the wsp mutations, resulting in overexpression of QSregulated extracellular products (proteases, exopolysaccharides). Why the lasR mutants did not emerge in the co-culture is unclear. Since las $R$ deficiency reduced the $S$. aureus killing activity of the $w s p F$ mutant, it would probably prevent $P$. aeruginosa from taking advantage of nutrients released by lysed $S$. aureus cells.

Lipopolysaccharide represents the main component of the outer cell membrane of Gram-negative bacteria and constitutes an important virulence factor. Most $P$. aeruginosa strains produce two chemically distinct forms of LPS, capped with common polysaccharide antigen and OSA, O-antigen respectively. In $P$. aeruginosa $\mathrm{PAO} 1$, the $\mathrm{WbpL}$ glycosyltransferase is involved in the synthesis of both the common polysaccharide antigen and OSA LPS (Murphy et al., 2014). Interestingly, PA14 does not produce the common polysaccharide antigen (Hao et al., 2015); thus, the loss of OSA leaves only uncapped LPS cores, a characteristic found in hostadapted clinical isolates of $P$. aeruginosa that chronically infect CF-patients (Folkesson et al., 2012). Loss of OSA was associated with fitness gain in the presence of $S$. aureus. Furthermore, this particular feature could explain the commensallike interactions between clinical isolates of $P$. aeruginosa and $S$. aureus (Michelsen et al., 2014). However, it does not provide an explanation for the selection of these mutants. At physiological $\mathrm{pH}$, bacterial cells of $P$. aeruginosa that express only the OSA LPS display a hydrophilic surface, whereas those expressing only the core LPS are more hydrophobic (Makin and Beveridge, 1996). This hydrophobicity of the bacterial cell surface could represent a defense mechanism to a substance produced by $S$. aureus targeting $P$. aeruginosa either directly, or indirectly by enhancing biofilm-formation or cell aggregation. Both the co-culture evolved strain (C15C3) harboring the $w b p L_{\mathrm{PA} 14}{ }^{*} \mathrm{D} 249 \mathrm{G}$ mutation, and a $\Delta w b p L_{\mathrm{PA} 14}$ mutant showed increased resistance to beta-lactams, which could be restored by extrachromosomal complementation with the $w b p L_{\mathrm{PA} 14}$ wild-type allele. Loss of LPS has been previously associated with an increased 
resistance to antibiotics and more specifically to small charged molecules (Alvarez-Ortega et al., 2010). Effects on the AmpC cephalosporinase expression or exopolysaccharide overexpression, occurring in the $w s p F$ mutant, could be possible alternative explanations. Taken together, LPS seems to play a role in the interactions between $P$. aeruginosa and $S$. aureus, although the precise nature of these interactions remains to be determined.

In conclusion, we showed that wsp mutants occurred in both mono- and co-cultures, and likely represent an adaptation to the oxygen-limiting conditions in our static in vitro evolution assay. Interestingly, all tested wsp mutations showed increased production of secreted proteins and probably other soluble factors, which have bactericidal activity on other Gram-negative and Grampositive bacterial species. Thus, selection of wsp mutants does not represent adaptation to the $S$. aureus population. In contrast, mutations in the LPS biosynthesis pathway emerged exclusively within the co-cultures. Mutations in $w b p L_{\mathrm{PA} 14}$ were present in all replicates, causing the loss of OSA-LPS leading to increased resistance towards beta-lactams. Whether LPS loss is a defense mechanism against $S$. aureus or a stealth-mode strategy requires further investigations. Strikingly, both $w s p F$ and LPSdeficient mutants are found among CF-isolates, suggesting that interspecies adaptation drives evolution in the CF-lung, which can also contribute to antibiotic resistance. Our study emphasizes that evolved interactions between bacterial species can be the result of both direct selection imposed by interacting species and a correlated response to selection imposed by other aspects of the environment.

\section{Conflict of Interest}

The authors declare no conflict of interest.

\section{Acknowledgements}

This study was supported by the Swiss National Science Foundation (grants $n^{\circ} 140929$ and $n^{\circ} 159523$ ). Research performed in the laboratory of JSL is supported by the Canadian Institutes of Health Research (grant MOP-14687). The authors would like to acknowledge Julien Prados for his help with the evolved genomes assembly and the mutation analysis. $\mathrm{YH}$ is a recipient of a postdoctoral fellowship from Cystic Fibrosis Canada. AB is supported by the Royal Society and J.S.L. holds a Canada Research Chair in Cystic Fibrosis and Microbial Glycobiology.

\section{References}

Abdel-Mawgoud AM, Lepine F, Deziel E. (2010). Rhamnolipids: diversity of structures, microbial origins and roles. Appl Microbiol Biotechnol 86: 1323-1336.
Alvarez-Ortega C, Wiegand I, Olivares J, Hancock RE, Martinez JL. (2010). Genetic determinants involved in the susceptibility of Pseudomonas aeruginosa to betalactam antibiotics. Antimicrob agents chemother 54: 4159-4167.

Arevalo-Ferro C, Hentzer M, Reil G, Gorg A, Kjelleberg S, Givskov $\mathrm{M}$ et al. (2003). Identification of quorumsensing regulated proteins in the opportunistic pathogen Pseudomonas aeruginosa by proteomics. Environ Microbiol 5: 1350-1369.

Baldan R, Cigana C, Testa F, Bianconi I, De Simone M, Pellin D et al. (2014). Adaptation of Pseudomonas aeruginosa in cystic fibrosis airways influences virulence of Staphylococcus aureus in vitro and murine models of co-infection. PLoS One 9: e89614.

Bantinaki E, Kassen R, Knight CG, Robinson Z, Spiers AJ, Rainey PB. (2007). Adaptive divergence in experimental populations of Pseudomonas fluorescens. III. Mutational origins of wrinkly spreader diversity. Genetics 176: 441-453.

Cooper VS, Staples RK, Traverse CC, Ellis CN. (2014). Parallel evolution of small colony variants in Burkholderia cenocepacia biofilms. Genomics 104: 447-452.

Cystic Fibrosis Foundation Patient Registry. (2015). Annual data report. Bethesda, MD, USA.

DeLeon S, Clinton A, Fowler H, Everett J, Horswill AR, Rumbaugh KP. (2014). Synergistic interactions of Pseudomonas aeruginosa and Staphylococcus aureus in an in vitro wound model. Infect Immun $\mathbf{8 2}$ : 4718-4728.

Diaz De Rienzo MA, Stevenson PS, Marchant R, Banat IM. (2016). Effect of biosurfactants on Pseudomonas aeruginosa and Staphylococcus aureus biofilms in a BioFlux channel. Appl Microbiol Biotechnol 100: 5773-5779.

Folkesson A, Jelsbak L, Yang L, Johansen HK, Ciofu O, Hoiby $\mathrm{N}$ et al. (2012). Adaptation of Pseudomonas aeruginosa to the cystic fibrosis airway: an evolutionary perspective. Nature rev Microbiol 10: 841-851.

Frydenlund Michelsen C, Hossein Khademi SM, Krogh Johansen H, Ingmer H, Dorrestein PC, Jelsbak L. (2016). Evolution of metabolic divergence in Pseudomonas aeruginosa during long-term infection facilitates a proto-cooperative interspecies interaction. ISME j 10: 1323-1336.

Goymer P, Kahn SG, Malone JG, Gehrig SM, Spiers AJ, Rainey PB. (2006). Adaptive divergence in experimental populations of Pseudomonas fluorescens. II. Role of the GGDEF regulator WspR in evolution and development of the wrinkly spreader phenotype. Genetics 173: $515-526$.

Guvener ZT, Harwood CS. (2007). Subcellular location characteristics of the Pseudomonas aeruginosa GGDEF protein, WspR, indicate that it produces cyclic-di-GMP in response to growth on surfaces. Mol Microbiol 66: 1459-1473.

Hao Y, Murphy K, Lo RY, Khursigara CM, Lam JS. (2015). Single-nucleotide polymorphisms found in the migA and wbpX glycosyltransferase genes account for the intrinsic lipopolysaccharide defects exhibited by Pseudomonas aeruginosa PA14. J bacteriol 197: 2780-2791.

Harrison F, Paul J, Massey RC, Buckling A. (2008). Interspecific competition and siderophore-mediated cooperation in Pseudomonas aeruginosa. ISME $j$ 2: 49-55. 
Haussler S, Fuqua C. (2013). Biofilms 2012: new discoveries and significant wrinkles in a dynamic field. J Bacteriol 195: 2947-2958.

Hickman JW, Tifrea DF, Harwood CS. (2005). A chemosensory system that regulates biofilm formation through modulation of cyclic diguanylate levels. Proc Natl Acad Sci USA 102: 14422-14427.

Hoffman LR, Deziel E, D'Argenio DA, Lepine F, Emerson J, McNamara S et al. (2006). Selection for Staphylococcus aureus small-colony variants due to growth in the presence of Pseudomonas aeruginosa. Proc Natl Acad Sci USA 103: 19890-19895.

Jimenez PN, Koch G, Thompson JA, Xavier KB, Cool RH, Quax WJ. (2012). The multiple signaling systems regulating virulence in Pseudomonas aeruginosa. Microbiol Mol Biol Rev 76: 46-65.

Kessler E, Safrin M, Olson JC, Ohman DE. (1993). Secreted LasA of Pseudomonas aeruginosa is a staphylolytic protease. J Biol Chem 268: 7503-7508.

Kim S, Yoon Y, Choi KH. (2015). Pseudomonas aeruginosa DesB promotes Staphylococcus aureus growth inhibition in coculture by controlling the synthesis of HAQs. PLoS One 10: e0134624.

Kirisits MJ, Parsek MR. (2006). Does Pseudomonas aeruginosa use intercellular signalling to build biofilm communities? Cell Microbiol 8: 1841-1849.

Köhler T, Buckling A, van Delden C. (2009). Cooperation and virulence of clinical Pseudomonas aeruginosa populations. Proc Natl Acad Sci USA 106: 63396344.

Koza A, Moshynets O, Otten W, Spiers AJ. (2011). Environmental modification and niche construction: developing $\mathrm{O} 2$ gradients drive the evolution of the Wrinkly Spreader. ISME J 5: 665-673.

Li H, Durbin R. (2010). Fast and accurate long-read alignment with Burrows-Wheeler transform. Bioinformatics 26: 589-595.

Makin SA, Beveridge TJ. (1996). The influence of A-band and B-band lipopolysaccharide on the surface characteristics and adhesion of Pseudomonas aeruginosa to surfaces. Microbiology 142(Pt 2): 299-307.

Malone JG. (2015). Role of small colony variants in persistence of Pseudomonas aeruginosa infections in cystic fibrosis lungs. Infect Drug Resist 8: 237-247.

Marquart ME, Caballero AR, Chomnawang M, Thibodeaux BA, Twining SS, O'Callaghan RJ. (2005). Identification of a novel secreted protease from Pseudomonas aeruginosa that causes corneal erosions. Invest Ophthalmol Vis Sci 46: 3761-3768.

Marvig RL, Johansen HK, Molin S, Jelsbak L. (2013). Genome analysis of a transmissible lineage of pseudomonas aeruginosa reveals pathoadaptive mutations and distinct evolutionary paths of hypermutators. PLoS genet 9: e1003741.

Marvig RL, Sommer LM, Molin S, Johansen HK. (2015). Convergent evolution and adaptation of Pseudomonas aeruginosa within patients with cystic fibrosis. Nat Genet 47: 57-64.

Mashburn LM, Jett AM, Akins DR, Whiteley M. (2005). Staphylococcus aureus serves as an iron source for Pseudomonas aeruginosa during in vivo coculture. J bacteriol 187: 554-566.
Michelsen CF, Christensen AM, Bojer MS, Hoiby N, Ingmer H, Jelsbak L. (2014). Staphylococcus aureus alters growth activity, autolysis, and antibiotic tolerance in a human host-adapted Pseudomonas aeruginosa lineage. J bacteriol 196: 3903-3911.

Murphy K, Park AJ, Hao Y, Brewer D, Lam JS, Khursigara CM. (2014). Influence of $\mathrm{O}$ polysaccharides on biofilm development and outer membrane vesicle biogenesis in Pseudomonas aeruginosa PAO1. J bacteriol 196: 1306-1317.

O'Rourke D, FitzGerald CE, Traverse CC, Cooper VS. (2015). There and back again: consequences of biofilm specialization under selection for dispersal. Front Genet 6: 18.

Rocchetta HL, Burrows LL, Pacan JC, Lam JS. (1998). Three rhamnosyltransferases responsible for assembly of the A-band D-rhamnan polysaccharide in Pseudomonas aeruginosa: a fourth transferase, $\mathrm{WbpL}$, is required for the initiation of both A-band and B-band lipopolysaccharide synthesis. Mol Microbiol 28: 1103-1119.

Rudin L, Sjostrom JE, Lindberg M, Philipson L. (1974). Factors affecting competence for transformation in Staphylococcus aureus. J bacteriol 118: 155-164.

Simpson JT, Durbin R. (2010). Efficient construction of an assembly string graph using the FM-index. Bioinformatics 26: i367-i373.

Smith EE, Buckley DG, Wu Z, Saenphimmachak C, Hoffman LR, D'Argenio DA et al. (2006). Genetic adaptation by Pseudomonas aeruginosa to the airways of cystic fibrosis patients. Proc Natl Acad Sci USA 103: 8487-8492.

Spiers AJ, Kahn SG, Bohannon J, Travisano M, Rainey PB. (2002). Adaptive divergence in experimental populations of Pseudomonas fluorescens. I. Genetic and phenotypic bases of wrinkly spreader fitness. Genetics 161: 33-46.

Spiers AJ, Bohannon J, Gehrig SM, Rainey PB. (2003). Biofilm formation at the air-liquid interface by the Pseudomonas fluorescens SBW25 wrinkly spreader requires an acetylated form of cellulose. Mol Microbiol 50: $15-27$.

Starkey M, Hickman JH, Ma L, Zhang N, De Long S, Hinz A et al. (2009). Pseudomonas aeruginosa rugose smallcolony variants have adaptations that likely promote persistence in the cystic fibrosis lung. J bacteriol 191: 3492-3503.

Wang S, Hao Y, Lam JS, Vlahakis JZ, Szarek WA, Vinnikova A et al. (2015). Biosynthesis of the common polysaccharide antigen of Pseudomonas aeruginosa PAO1: characterization and role of GDP-D-Rhamnose: GlcNAc/GalNAc-diphosphate-lipid alpha1,3-D-rhamnosyltransferase WbpZ. J bacteriol 197: 2012-2019.

Wiser MJ, Lenski RE. (2015). A comparison of methods to measure fitness in Escherichia coli. PLoS One 10: $\mathrm{e} 0126210$.

Yang L, Liu Y, Markussen T, Hoiby N, Tolker-Nielsen T, Molin S. (2011). Pattern differentiation in co-culture biofilms formed by Staphylococcus aureus and Pseudomonas aeruginosa. FEMS Immunol Med Microbiol 62: 339-347.

Supplementary Information accompanies this paper on The ISME Journal website (http://www.nature.com/ismej) 